\title{
Preliteracy signatures of poor-reading abilities in resting-state EEG
}

\section{Giuseppina Schiavone ${ }^{1,2}$, Klaus Linkenkaer-Hansen ${ }^{1 *}$, Natasha M. Maurits ${ }^{3}$, Anna Plakas ${ }^{3,4}$, Ben A. M. Maassen ${ }^{5}$, Huibert D. Mansvelder ${ }^{1}$, Aryan van der Leij ${ }^{4}$ and Titia L. van Zuijen ${ }^{4}$}

${ }^{1}$ Department of Integrative Neurophysiology, Center for Neurogenomics and Cognitive Research, VU University Amsterdam, Amsterdam, Netherlands

2 Body Area Network, imec/Holst Centre, Eindhoven, Netherlands

${ }^{3}$ Department of Neurology, University Medical Center Groningen, University of Groningen, Groningen, Netherlands

${ }^{4}$ Research Institute of Child Development and Education, University of Amsterdam, Amsterdam, Netherlands

${ }^{5}$ Center for Language and Cognition Groningen and University Medical Center Groningen, University of Groningen, Groningen, Netherlands

\section{Edited by:}

Donatella Spinelli, Università di

Roma Foro Italico, Italy

Reviewed by:

Satu Palva, University of Helsinki,

Finland

Chiara Spironelli, Department of

General Psychology, Italy

\section{*Correspondence:}

Klaus Linkenkaer-Hansen,

Department of Integrative

Neurophysiology, Center for

Neurogenomics and Cognitive

Research, VU University

Amsterdam, Neuroscience Campus

Amsterdam, De Boelelaan 1085,

1081 HV Amsterdam, Netherlands

e-mail:klaus.linkenkaer@cncr.vu.nl
The hereditary character of dyslexia suggests the presence of putative underlying neural anomalies already in preliterate age. Here, we investigated whether early neurophysiological correlates of future reading difficulties-a hallmark of dyslexia-could be identified in the resting-state EEG of preliterate children. The children in this study were recruited at birth and classified on the basis of parents' performance on reading tests to be at-risk of becoming poor readers $(n=48)$ or not $(n=14)$. Eyes-open rest EEG was measured at the age of 3 years, and the at-risk children were divided into fluent readers $(n=24)$ and non-fluent readers $(n=24)$ after reading assessment at their third grade of school. We found that fluent readers and non-fluent readers differed in normalized spectral amplitude. Non-fluent readers were characterized by lower amplitude in the delta- 1 frequency band $(0.5-2 \mathrm{~Hz})$ and higher amplitude in the alpha- 1 band $(6-8 \mathrm{~Hz})$ in multiple scalp regions compared to control and at-risk fluent readers. Interestingly, across groups these EEG biomarkers correlated with several behavioral test scores measured in the third grade. Specifically, the performance on reading fluency, phonological and orthographic tasks and rapid automatized naming task correlated positively with delta-1 and negatively with alpha-1. Together, our results suggest that combining family-risk status, neurophysiological testing and behavioral test scores in a longitudinal setting may help uncover physiological mechanisms implicated with neurodevelopmental disorders such as the predisposition to reading disabilities.

Keywords: precursors of reading disabilities, resting-state EEG, reading fluency, delta and alpha oscillations

\section{INTRODUCTION}

Dyslexia is a learning disorder that specifically impairs a child's technical reading ability. It affects about $5-10 \%$ of all children, with higher prevalence in families with one or more members having dyslexia (Lyon et al., 2003). Dyslexia cannot be explained by low intelligence, low-level vision, hearing impairments, or poor education, but phonological deficits and problems with rapid automatized naming are commonly observed readingrelated deficits (Snowling et al., 2003; van Bergen et al., 2012). These reading impairments affect the ability to read fluently and are often a cause of frustration and distress for a child, producing severe social and psychological consequences in their lifespan (Vellutino and Scanlon, 1987; Humphrey and Mullins, 2004).

Several genes involved in early brain development have been suggested to cause susceptibility to dyslexia (Scerri and SchulteKorne, 2010). Thus, even though dyslexia manifests in school years, underlying neural anomalies may already be present in the preliterate brain. This is supported by a number of longitudinal studies using auditory event-related potentials (Molfese, 2000; Guttorm et al., 2001; Maurer et al., 2003, 2009; Lyytinen et al., 2004; Van Zuijen et al., 2012, 2013) and visual event-related potentials (Regtvoort et al., 2006; Schulte-Korne and Bruder, 2010; Araujo et al., 2012) reporting differences in brain responses to reading-related stimuli (e.g., speech sounds or visual contrast) between familial risk and control groups, or between control children and children that later become poor readers.

In adults or school-aged children differences between dyslexics and typical readers have been reported in ongoing EEG activity. Comparing these two groups has pointed to higher delta and theta activity during phonological task (Rippon, 2000; Klimesch et al., 2001a; Spironelli et al., 2006; Penolazzi et al., 2008) and lower alpha and beta activity (Rumsey et al., 1989; Rippon, 2000; Klimesch et al., 2001b) during reading tasks in dyslexics. Several studies (Sklar et al., 1972; Colon et al., 1979; Ahn et al., 1980; Duffy et al., 1980; Pinkerton et al., 1989; Rumsey et al., 1989; Harmony et al., 1995; Clarke et al., 2002; Benasich et al., 2008; Gou et al., 2011; Babiloni et al., 2012) have also investigated EEG from the resting-state. However, differences in participant cohorts-ranging from preschool or school-age children, to adolescents or adults and varying degrees of language disability—and 
in EEG biomarker definitions has prevented a consensus about possible neuronal signatures of dyslexia.

Throughout development neuronal oscillations plays an important role in shaping the structural and functional neuronal connectivity that will support higher brain functions, such as language and cognition, later in life (Smit et al., 2011). Early impairments in resting-state EEG, reflecting underling neuronal activation, might prelude future developmental problems. In line with these considerations and previous findings, we test whether ongoing neuronal oscillations in preliterate children carry information about reading fluency later in life. We compared relative amplitude spectra of EEG measured at about 3 years of age in three groups of children: one control group of fluent readers and two at-risk groups of fluent and non-fluent readers. Reading fluency was assessed at third grade of school, when the children were about 9 years old. We identified two EEG biomarkers that correlated with reading performance and reading-related test scores collected in third grade.

\section{MATERIALS AND METHODS SUBJECTS AND SELECTION CRITERIA}

The children that participated in this study are part of the Dutch Dyslexia Programme, a longitudinal research project. The study was approved by the medical ethics committee of the University of Nijmegen, the Netherlands. Informed consent was obtained from one of the parents of each child. Parents were recruited when expecting a baby. The children were first divided in control and at-risk groups, then the at-risk group was divided in fluent and non-fluent readers.

To assess whether the infants were at familial risk of becoming poor readers, the reading fluency of the parents was tested with a word reading task (Brus and Voeten, 1973) and a pseudoword reading task (Van den Bos et al., 1994). In addition, verbal reasoning was measured with the subtest Similarities of the Wechsler Adult Intelligence Scale (Wechsler, 1997; Dutch adaption: Uterwijk, 2000). Children were included in the at-risk group when one of the parents scored (1) lower or at the 20th percentile on both reading tests, (2) lower or at the 10th percentile on one reading test and below the 50th percentile on the other reading test, (3) lower than the 15th percentile on one reading test and not higher than the 40th percentile on the other reading test, or (4) with a discrepancy of 60 percentiles or more between the verbal reasoning test and either of the two reading tests and the additional requirement that both reading scores were below the 50th percentile. The children were included in the control group when both parents scored at the 50th percentile or higher on both reading tests.

Reading in the children was assessed based on three measurements: at the beginning of second grade (2.4 months after starting second grade, SD 1.7 months; the children were 7 years and 6 months of age, SD 5.1 months) with two word-reading lists (1A and $1 \mathrm{~B}$ from the 3-min test, Verhoeven, 1995), at the end of second grade (6.0 months after starting second grade, SD 0.9 month) with a word reading list (2A from the 3 min test, Verhoeven, 1995) and a pseudoword reading list (Van den Bos et al., 1994), and in the middle of third grade (3.5 months after starting third grade, SD 1.1 month) with a word reading list (Brus and Voeten, 1973) and a pseudoword reading list (Van den Bos et al., 1994). A child was classified as a "non-fluent reader" when it scored poor on two out of three measurements. A child was marked "poor" when it scored below or at the 10th percentile on one of the reading lists and below the 50th percentile on the other reading list, or below or at the 25th percentile on both reading lists. Two children, initially selected as part of the control group, scored as "non-fluent readers" and were excluded from the analysis. One child that was selected to be part of the at-risk group showed general cognitive delay and was omitted as well. This resulted in three groups: a control group of fluent readers ( $\mathrm{C}, 14$ children, 9 boys), a group of at-risk fluent readers (RF, 24 children, 16 boys) and a group of at-risk non-fluent readers (RNF, 24 children, 14 boys).

\section{BEHAVIORAL EVALUATION}

Children were submitted to a range of cognitive tests that were administered in the middle of third grade. Two subtests of the Wechsler Intelligence Scale for children (WISC-III, Wechsler, 1992; Dutch adaptation: Kort et al., 2002) were administered: the subtest Block Design measuring nonverbal visual-spatial skills and the subtest Vocabulary measuring expressive vocabulary. Two children from the at-risk fluent group and one child from the at-risk non-fluent group were absent during this evaluation; therefore, they were not included in the behavioral statistical analysis. Behavioral tests for assessing reading-related skills were the rapid automatized naming task (RAN, Van den Bos et al., 1994), a phoneme deletion task (Amsterdamse klankdeletietest, AKT, De Jong and Van der Leij, 2003) and an orthographic choice task (Horsley, 2005). RAN measures the speed of naming overlearned information. The child was requested to name 50 digits from a piece of paper. The naming time was measured and the score was then expressed in the number of digits a child could have named in a minute. The phoneme deletion task measures phonological awareness. The child was asked to repeat a pseudoword (e.g., "memslos"), and subsequently asked to leave out a specific phoneme (e.g., the sound "l"), and to pronounce the resulting word ("memsos"). The score is the number of correct items out of 27. The orthographic choice task measured orthographic knowledge. The child had to decide the correct spelling of a word presented together with two homophonic pseudowords (e.g., among "vurkeer, verkeer, verkir" the correct spelling is "verkeer"). The score was the number of correct answers out of 70 items that the child completed in $10 \mathrm{~min}$.

\section{EEG RECORDING}

Neurophysiological data were collected at 35.1 months of age (SD 0.4 months). EEG was recorded from 64 channels (positioned according to the International 10-20 system; $500 \mathrm{~Hz}$ sampling rate; filtered at $0.01-100 \mathrm{~Hz}$ ), including mastoid references and vertical and horizontal electrooculogram (SynAmps2 64 Channel Quik-Cap, Neuroscan). Three to five min eyes-open rest EEG were collected while the child was on the parent's lap; the child was awake and was encouraged to look at moving lines on a screen to keep it sitting as still as possible. To ensure objectively similar artifact rejection across the different cohorts, the recorded EEG was filtered and cleaned offline using FASTER, a Matlab toolbox for automatic EEG artifacts rejection (for details, see 
Nolan et al., 2010). In brief, after filtering (0.4-30 Hz band-pass), FASTER segmented the signals into 1-s epochs, detected and interpolated noisy channels, removed contaminated epochs and ran Independent Components Analysis (ICA) for the identification and the rejections of ICs associated with EOG, EMG artifacts. Finally, the signals were re-referenced to the common average, i.e., the average of all remaining scalp electrodes. The mean number of interpolated channels was 2 ( $\pm 1 \mathrm{SD}$, standard deviation) and the mean distance between interpolated channels was in $86 \%$ of the cases greater than the distance between neighbor channels. Thus, the influence of the interpolation on the spatial density of scalp EEG was negligible. The duration of the artifact-free epochs in each recording was $1.9 \pm 0.1 \mathrm{~min}$ (mean \pm standard error).

\section{SPECTRAL ANALYSIS OF EEG DATA}

Artifact-free epochs were submitted to spectral analysis. Power spectral analysis was computed using Fast Fourier Transform (Welch technique, Hamming windowing function, with 4096 FFT points, resulting in frequency bins of width $0.1 \mathrm{~Hz}$ ). We observed multiple peaks in the $6-12 \mathrm{~Hz}$ range in all subjects and, therefore, we calculated the individual alpha frequency using the gravity frequency peak definition $\left(\sum \frac{\mathrm{a}(\mathrm{f}) \times \mathrm{f}}{\sum \mathrm{a}(\mathrm{f})}\right.$, with a (f) denoting the power spectral density at frequency $f$, and $\sum()$ the sum computed over the frequency bins in the interval $6-12 \mathrm{~Hz}$ ) (Klimesch, 1999). Mean gravity frequency peak across central electrodes $(\mathrm{C} 1, \mathrm{Cz}, \mathrm{C} 2, \mathrm{CP} 1, \mathrm{CPz}, \mathrm{CP} 2)$ was obtained for each subject in each group (mean and standard error of mean for C: $8 \pm 0.04 \mathrm{~Hz}$; for RF: $8 \pm 0.04 \mathrm{~Hz}$; for RNF: $7.9 \pm 0.07 \mathrm{~Hz}$ ). No statistically significant differences were found between the groups $\left[F_{(2,59)}=1.17 ; p=0.3\right.$, ANOVA $]$; similarly, no differences for gender $\left[F_{(1,60)}=1.06 ; p=0.3\right.$, ANOVA $]$ or for the interaction group $\times$ gender were found $\left[F_{(2,56)}=0.4 ; p=0.7\right.$, ANOVA]. Given these results we considered a common individual alpha frequency of $8 \mathrm{~Hz}$ (consistent with literature findings in this age range Stroganova et al., 1999; Marshall et al., 2002) and we defined the frequency bands accordingly (Klimesch, 1999; Babiloni et al., 2012): 0.5-2 Hz (delta-1), 2-4 Hz (delta-2), 4-6 Hz (theta), 6-8 Hz (alpha-1), 8-10 Hz (alpha-2), 10-13 Hz (alpha3), 13-20 Hz (beta-1), 20-30 Hz (beta-2). The amplitude of EEG signals depends on several factors unrelated to neuro-electrical activity such as anatomical and physical properties of the brain and surrounding tissue (bone thickness, skull resistance and impedance). These parameters vary from one subject to another; however, their influence on the statistical analysis can be minimized by the use of relative amplitude spectra, because these factors equally affect all frequencies analyzed. Amplitude spectra for each electrode were computed as the square root of the power spectra. The relative amplitude spectra were obtained by normalizing as follows:

$$
\frac{\left\langle P_{B_{i}}\right\rangle}{\sum_{i=1}^{n}\left\langle P_{B_{i}}\right\rangle}
$$

where, \langle\rangle indicates the average of the amplitude in a specific frequency band, $\mathrm{P}_{\mathrm{B}_{\mathrm{i}}}$, across frequency bins, and $i=1: \mathrm{n}$, with $n=8$, corresponds to the ith frequency band considered in the analysis. The relative amplitude in these eight frequency bands were computed with the NBT toolbox (www.nbtwiki.net) (Hardstone et al., 2012), and are referred to as "biomarkers" following the broad definition: "A characteristic that is objectively measured and evaluated as an indicator of normal biological processes, pathogenic processes, or pharmacological responses to a therapeutic intervention" (Frank and Hargreaves, 2003).

\section{STATISTICAL ANALYSIS FOR BEHAVIORAL DATA}

The behavioral tests of word and pseudoword reading, the nonverbal and verbal intelligence subtests, phoneme awareness test, orthographic knowledge test and the RAN tasks were evaluated by One-Way ANOVAs in order to determine differences among the three groups. Tukey's post-hoc test was used for post-hoc analysis with multiple comparison correction. For correlation analysis with EEG biomarkers, reading fluency performance scores were defined as a composite measure of performance in the two reading tasks measured at the middle of the third grade: word reading and pseudoword reading. Reading fluency performance scores were computed as the $\mathrm{z}$-scores of the average $\mathrm{z}$-scores of the performance in two reading tasks.

\section{STATISTICAL ANALYSIS OF EEG DATA}

Distributions of amplitude spectra in each group for each channel and frequency band were tested for normality with the Lilliefors test. For about $25 \%$ of the channels the null hypothesis of normal distribution was rejected $(p<0.05)$; for this reason non-parametric methods were used for the statistical analysis. Statistical analysis of differences between groups was performed with non-parametric One-Way ANOVA (Kruskal-Wallis test) with group (C, RF, RNF) as main factor for each electrode and frequency band. Wilcoxon rank-sum test for each channel was used for between-group comparison and to compare means across significant electrodes. As an alternative to multiple comparison correction, we performed binomial testing to validate the statistical significance of the results (Maris and Oostenveld, 2007; Montez et al., 2009; Nikulin et al., 2012); i.e., differences were only considered significant if at least 10 electrodes would reach a $p$-value below 0.05 . The likelihood of having this many channels out of 64 reach a $p$-value below 0.05 by chance is less than $0.1 \%$ (cf., binomial distribution). No correction was applied across frequency bands.

\section{CORRELATION ANALYSIS BETWEEN BEHAVIORAL AND NEUROPHYSIOLOGICAL DATA}

The behavioral measures (the reading fluency scores, the scores on phonological awareness, orthographic knowledge, and RAN) that were all assessed in the middle of third grade were correlated with the mean amplitudes across significant channels of the most significant spectral bands using Spearman correlation. Correlation analysis was also performed for each channel as an alternative approach to identify brain regions with activity associated to later reading and reading-related skills.

\section{RESULTS}

\section{BEHAVIORAL RESULTS}

The three subject groups (control, C; at-risk fluent readers, RF; at-risk non-fluent readers, RNF) were assessed on a variety of reading and reading-related tests (see, Table $\mathbf{1}$ ). 
Table 1 | Behavioral measures.

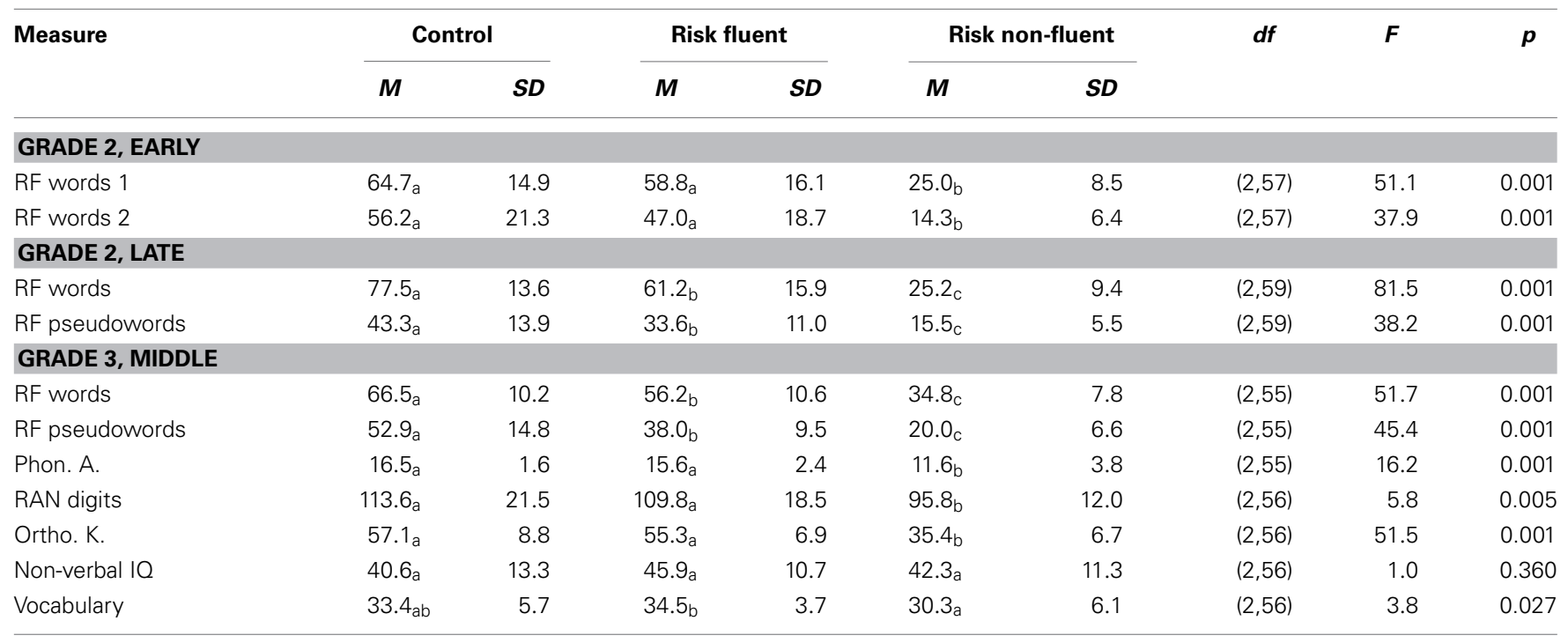

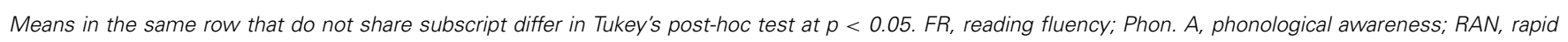
automatized naming; Orhto.K, orthographic knowledge.

The RNF children scored lower than the two fluent reading groups for phonological awareness (RNF vs. RF, $p=0.002$; RNF vs. $\mathrm{C}, p=0.001$ ), RAN digits (RNF vs. RF, $p=0.022$; RNF vs. C, $p=0.01$ ) and orthographic knowledge (RNF vs. RF, $p=0.001$; RNF vs. C, $p=0.001$ ) tasks. This confirms that non-fluent reading is accompanied by reading-related deficits in phonological awareness, rapid naming and orthographic knowledge (De Jong and Van der Leij, 2003; Vellutino et al., 2004).

The groups did not differ on the intelligence subtest of nonverbal IQ; however, the RNF readers scored lower than the RF readers in the vocabulary test $(p=0.024)$. This is a well-known effect and poor verbal abilities have been reported to be present already at preliteracy age in children later diagnosed with dyslexia studies (Snowling et al., 2003; van Bergen et al., 2013).

Performance on word and pseudoword reading fluency tests at the middle of the third grade differed between all groups with the control children performing best, followed by the at-risk fluent readers and the at-risk non-fluent readers (Table 1; for word reading: RNF vs. RF, $p=0.001$; RNF vs. $\mathrm{C}, p=0.001$; RF vs. $\mathrm{C}$, $p=0.007$; and for pseudoword reading: RNF vs. RF, $p=0.001$; RNF vs. $\mathrm{C}, p=0.001$; RF vs. $\mathrm{C}, p=0.001)$. This indicates that the RNF children had subclinical reading deficits possibly as a result of a higher liability (van Bergen et al., 2012).

\section{EEG CORRELATES OF FUTURE READING ABILITIES}

To investigate the presence of differences in preliteracy measures of brain function between those children who would later become fluent readers and those that would become non-fluent readers, we analyzed eyes-open rest EEG recorded at about 3 years of age using classical spectral analysis (Figure 1, and Materials and Methods). We performed an ANOVA to compare relative amplitude in eight different frequency bands among the three groups and observed marked differences in the delta- 1 and alpha1 bands (Figure 2). Electrodes with a significant effect formed spatially connected clusters over frontal and centro-parietal scalp regions for the delta- 1 band and over central and parieto-occipital regions for the alpha-1 band (see $p$-value topography maps in Figure 2, fourth column). For delta-1 we found 10 electrodes with a significant difference between groups at $p<0.05$, whereas 18 electrodes reached this level for alpha-1. According to binomial testing, the probability of obtaining an equal or larger number of electrodes than 10 was negligibly small $(p<1 \mathrm{e}-3$; Materials and Methods). On the contrary, for the theta and alpha-2 bands the number of significant electrodes was too small to reject the null hypothesis of the binomial significance test. The ANOVA for delta-2 did not show a main group effect at any electrode. No effects were observed in the higher frequency bands of alpha-3, beta-1, or beta-2 (data not shown). We note that alpha-2 oscillations displayed peaks bilaterally over the sensorimotor regions for all groups as previously reported for this age (Marshall and Meltzoff, 2011), which suggests that the alpha-1 result is not the child equivalent of the sensorimotor mu rhythm.

To identify more specifically the group differences that gave rise to the significant effects in the overall group comparisons we performed rank-sum tests between groups for delta- 1 and alpha1 relative amplitude. The results indicated that the control group did not differ from the at-risk fluent group in either of the two frequency bands (Figures 3A,B, first row), whereas widespread differences were observed for the comparisons of the control and at-risk non-fluent groups as well as for at-risk fluent and the atrisk non-fluent groups. Relative delta- 1 activity was low in at-risk non-fluent group compared to both the control and at-risk fluent group (Figure 3A, second and third row), whereas relative alpha1 activity was higher in the at-risk non-fluent group compared to both control and at-risk fluent group (Figure 3B, second and third row). The number of electrodes with $p<0.05$ in these comparisons varied between 7 and 33; the probability of obtaining an equal or larger number of electrodes for a binomial distribution was small ( $p<0.03$; Materials and Methods). Although one should be cautious in inferring the origin of the sources 
A

$$
\begin{aligned}
& \text { - Control } \\
& \text { - Risk Fluent } \\
& \text { - Risk Non Fluent }
\end{aligned}
$$

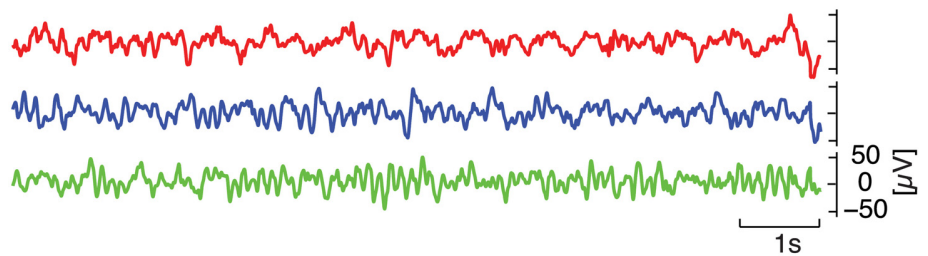

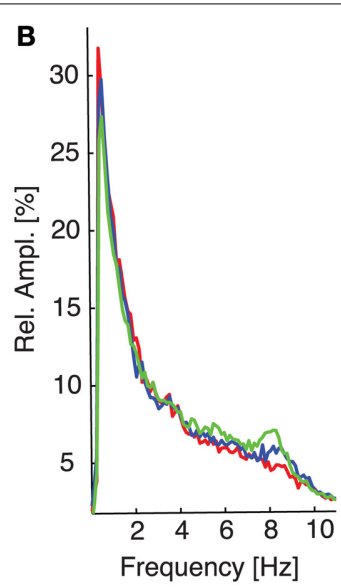

FIGURE 1 | EEG spectral analysis. (A) temporal evolution of $10 \mathrm{~s}$ of filtered signals $(0.4-30 \mathrm{~Hz})$ at the central electrode $(\mathrm{Cz})$ for three children belonging to each of the different groups (red line refers to control: blue line to at-risk fluent readers; green line to at-risk non-fluent readers). (B) medians of relative amplitude spectra in $\mathrm{Cz}$ across each group. giving rise to the scalp topographies shown in Figure 3, they could correspond to brain reading circuits identified in several neuroimaging studies to include parieto-temporal, occipito-temporal and inferior frontal lobes (Eckert et al., 2005; Richlan et al., 2009; Raschle et al., 2011). Together, these results indicate that children who became poor readers exhibited already at the age of three a peculiar resting-state EEG activity compared to both control and at-risk fluent groups.

\section{CORRELATION BETWEEN EEG BIOMARKERS AND BEHAVIORAL MEASURES}

Having identified relative amplitude of delta- 1 and alpha- 1 as putative biomarkers of future ability to read, we subsequently examined whether these EEG biomarkers could be related to performance in reading fluency and reading-related abilities (Figure 4, first row).

First, we computed the mean biomarker values of the delta-1 and alpha-1 across significant channels (see Figure 2, fifth column) and correlated these with the z-scores of the performance in the behavioral tests measured in the middle of the third grade. Within-group correlations did not reach statistical significance, which was expected given the restriction of range for the performance scores within groups. On the other hand, when considering all children together significant correlations were found between the EEG biomarkers and all the behavioral tests, i.e., Reading Fluency, Phonological Awareness, Orthographic Knowledge and RAN digits. Increasing performance on the different tasks correlated with increases in delta-1 amplitudes and decreases in alpha-1 amplitudes (correlation coefficients and $p$-values are reported in Figure 4, second row and third row). This result was to some extent expected given the group effects in the EEG and in the behavioral tests. Interestingly, however, when using the full range of correlations, scalp topographies revealed that also channels not showing a significant group difference were found to correlate with future performances (Figure 5).

Scalp topographies of correlations between the behavioral measures and the EEG biomarkers again suggested the involvement of multiple brain regions. In particular, delta1's relative amplitude correlations with Reading Fluency, with Phonological Awareness and with RAN digits appeared both in frontal, central, and parieto-temporal regions. Similar regions showed correlations with Orthographic Knowledge and, interestingly, also occipito-parietal regions showed a robust positive correlation (Figure 5, first row) with Orthographic Knowledge. For alpha-1's relative amplitude significant correlations were stronger in central sites and correlations with performance on the orthographic task were also observed in the occipital region (Figure 5, second row). Noteworthy, correlations over occipital sites were more evident in the orthographic task where processing of visual information takes place. Additionally, the correlations we have found over frontal and parietal electrodes for the phonological task might be in agreement with previous studies associating phonological processing with activity in frontal lobes and parietal and temporal regions (Buchsbaum, 2001; Burton, 2009).

\section{DISCUSSION}

The present study aimed at investigating neurophysiological correlates of later emerging reading abilities in the preliterate brain. Uniquely to our study, having longitudinal data covering about 9 years of development, we showed that characteristic spectral pattern in the resting-state EEG activity of children at 3 years of age discriminate children that became poor readers from children that became fluent readers. The ability to read fluently develops gradually over time and through substantial practice but in dyslexics it is hampered by the presence of reading-related deficits in phonological processing, mapping phonemes to graphemes, and automatic word recognition. Our behavioral results confirm this effect in poor readers showing that at-risk non-fluent children scored lower than both at-risk fluent and control children in all reading-related tests (Phonological Awareness, Orthographical Knowledge and RAN). Our data on intelligence measures were in agreement with previous findings showing that dyslexics score slightly lower on verbal tasks despite having adequate reasoning 


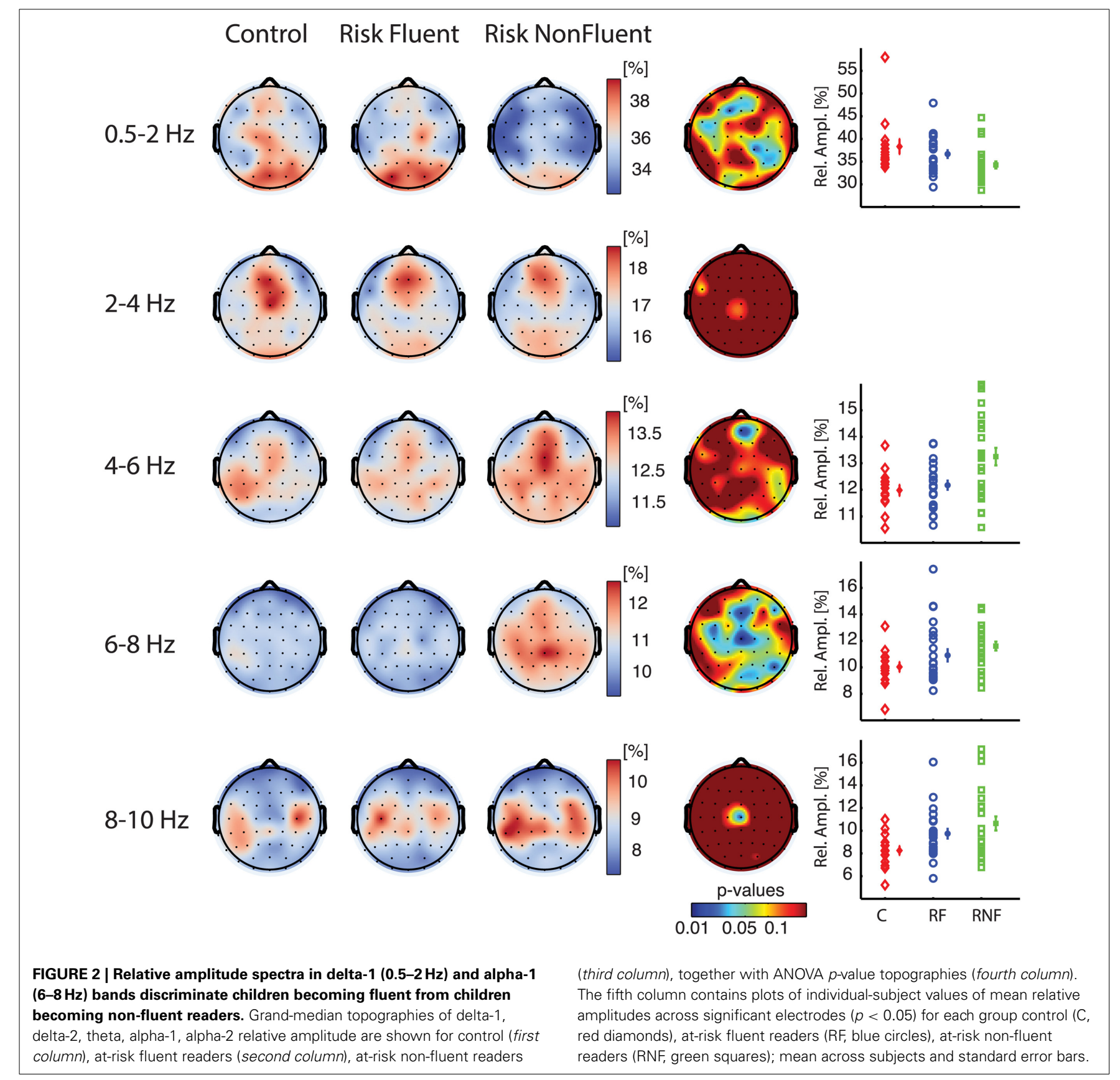

abilities compared to non-impaired readers (Snowling et al., 2003; van Bergen et al., 2013).

The comparison of EEG relative amplitude between the three groups of children, divided on the basis of their family-risk status and their reading fluency abilities, revealed several interesting findings. Our results show that two EEG biomarkers, delta-1 and alpha-1 relative amplitudes, emerged as putative preliterate discriminants of those children with a familiar risk of dyslexia who did become non-fluent readers. Non-fluent readers exhibited significantly lower levels of delta-1 activity and significantly higher alpha-1 activity compared to fluent readers (both control and atrisk fluent readers). Noteworthy, although the alpha-1 effect was prominent in central regions, we believe this does not reflect a sensorimotor mu rhythm deficit, because of the larger amplitude and clear bi-lateral topographic distribution of relative amplitude seen in alpha-2 band (Marshall and Meltzoff, 2011). Topographic distributions of the main group effect and of the correlations between the EEG biomarkers and behavioral data suggested the involvement of several scalp regions, which is in line with neuroimaging studies that have identified reading circuits both in parieto-temporal, occipito-temporal and inferior frontal lobes, albeit often with a left laterality (Eckert et al., 2005; Richlan et al., 2009; Raschle et al., 2011). Given that our statistical analysis was performed in sensor space, we are cautious with the interpretation 


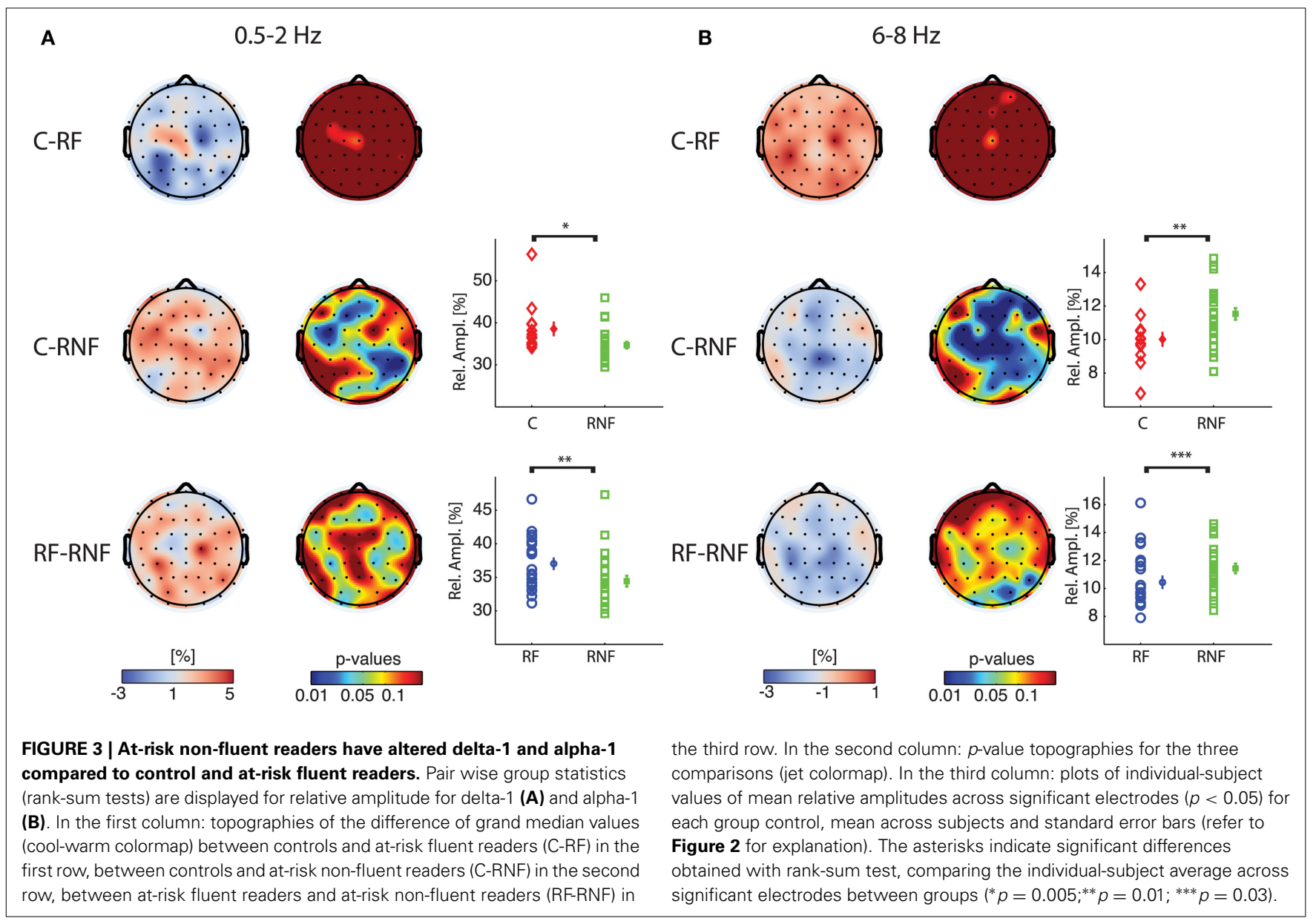

of the source origin of the effects shown in Figures 2, 3, 5; however, we note that both delta- 1 and alpha- 1 relative amplitude exhibited strong correlations with Orthographic Knowledge in occipital areas, which have previously been associated with orthographic processing (Samuelsson, 2000). Differently, phonological processing is known to involve frontal areas (Burton, 2009), which could explain the prominent effects we have found in the frontal scalp regions, especially for the delta-1. These results might suggest a broader role of delta activity and a specificity of alpha-1 oscillations for visual processing, present even before the reading onset.

\section{THE FUNCTION OF DELTA AND ALPHA OSCILLATIONS IN BRAIN MATURATION}

It is well known that delta activity dominates the human EEG during early development and decreases over the course of normal development (John et al., 1980; Gasser et al., 1988; Harmony et al., 1990). Slow wave delta activity in development is believed to be important in pruning redundant cortical connections and supports brain maturation as reflected in the positive association between delta activity and gray matter volume (Whitford et al., 2007). If slow-wave delta activity is indeed a necessary mechanism for pruning and cortical development to take place, our finding of reduced delta- 1 activity seem to be in agreement with the hypothesis of a cerebral maturation delay in 3-year old children that

later on become poor readers. Later in life, delta waves remain dominant during slow-wave sleep; however, a relatively high delta activity in a wakeful state has been associated with pathological neuronal conditions (Spironelli and Angrilli, 2009; Babiloni et al., 2012) such as in adults with ADHD and dyslexia (Chabot et al., 2001; Penolazzi et al., 2008). Higher delta activity has also been observed in dyslexic school-age children (Spironelli et al., 2006; Penolazzi et al., 2008; Spironelli and Angrilli, 2010) and dyslexic young adults (Rippon, 2000), although these data were recorded during reading tasks. However, increased delta and theta activity in dyslexics or children with reading and writing disabilities have also been reported in resting-state EEG at the age of 918 years (Sklar et al., 1972; Colon et al., 1979; Pinkerton et al., 1989; Harmony et al., 1995). Thus, in line with the hypothesis of dysfunctional development (Spironelli et al., 2006, 2010, 2011; Penolazzi et al., 2008), it is plausible that failure to produce adequate delta at a young age is part of the mechanism causing a delay of cortical maturation, which in turn may be reflected in an increase of delta activity in at-risk non-fluent readers compared to fluent readers at school-age. To investigate this, future analyses will be done on resting-state EEG collected in the present cohort at the age of 11 years.

In the present study, we used relative amplitude measures to reduce the considerable genetic variance on oscillatory power (Linkenkaer-Hansen et al., 2007). Thus, it is plausible that our 


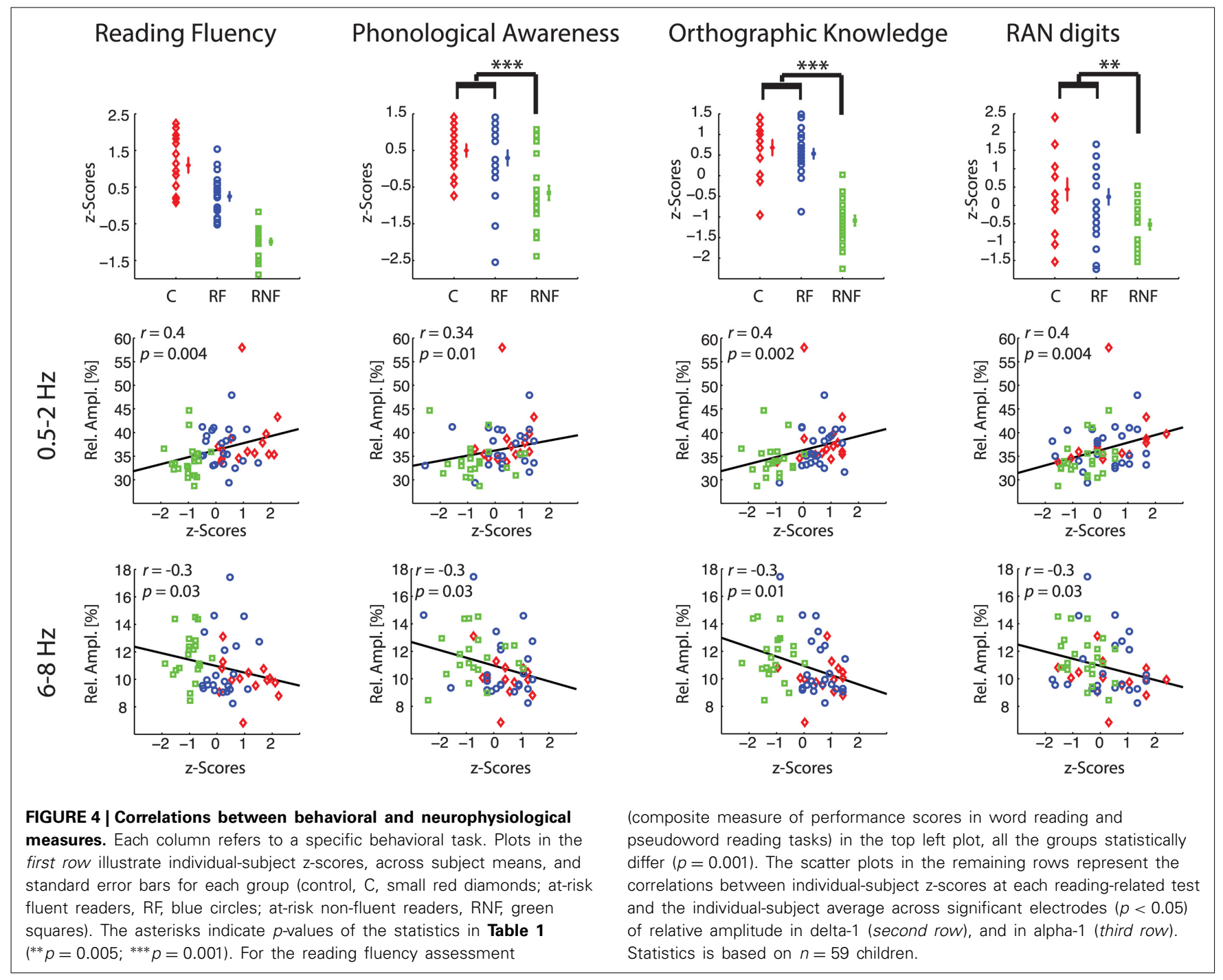

findings in the alpha-1 bands are to some extent related to those in the delta band. On the other hand, increases of lower alpha (spectral component just below the IAF) activity have been associated with difficulty in sustaining attention and inhibiting distracting environmental stimuli (Klimesch et al., 2001b). Thus, we cannot rule out that the children in our study differed in the level of attention paid to the moving lines, with the non-fluent reading group exhibiting low sustained attention, as reflected in higher alpha-1 amplitude compare to the other two groups.

\section{COMPARISON WITH OTHER FAMILIAL RISK STUDIES}

It is worth noting that our cohort resembles the cohort of the studies presented in Benasich et al. (2008) and Gou et al. (2011) where resting-state EEG of age-matched children (16, 24 , and 36 months of age) with a family history of languagebased learning impairment (LLI, FH+) and controls ( $\mathrm{FH}-$ ) were compared. Benasich et al. (2008) performed qualitative analysis among absolute power spectra in 9 frequency bins ranging from 5 to $50 \mathrm{~Hz}$ (excluding frequency components of the delta range) and across different scalp regions. They selected frontal and prefrontal regions and focused on two wide frequency ranges for their statistical analysis $(5-30 \mathrm{~Hz}$ and $31-50 \mathrm{~Hz}$, of which the latter was referred to as gamma). They reported lower frontal gamma power in $\mathrm{FH}+$ compared to $\mathrm{FH}-$, and found correlations of gamma power with attention, and expressive and receptive language skills measured at the same age. In a later study, using the same cohort of children, Gou et al. (2011) reported that resting frontal gamma power at 16, 24, and 36 months was associated with phonological memory and syntactical skill measured at the age of 4 and 5 years. Comparison with the present study are not possible for several reasons: (1) the definition of the family risk in our study is based on the parents' performance on reading tests, whereas in Benasich et al. (2008) it is associated to the presence of at least one sibling or parent diagnosed with LLI (75\% were siblings Choudhury and Benasich, 2003); (2) none of the children in Gou et al. (2011) were themselves diagnosed with any language or learning disabilities, whereas our at-risk children were divided into groups that became fluent or non-fluent readers; (3) the EEG 


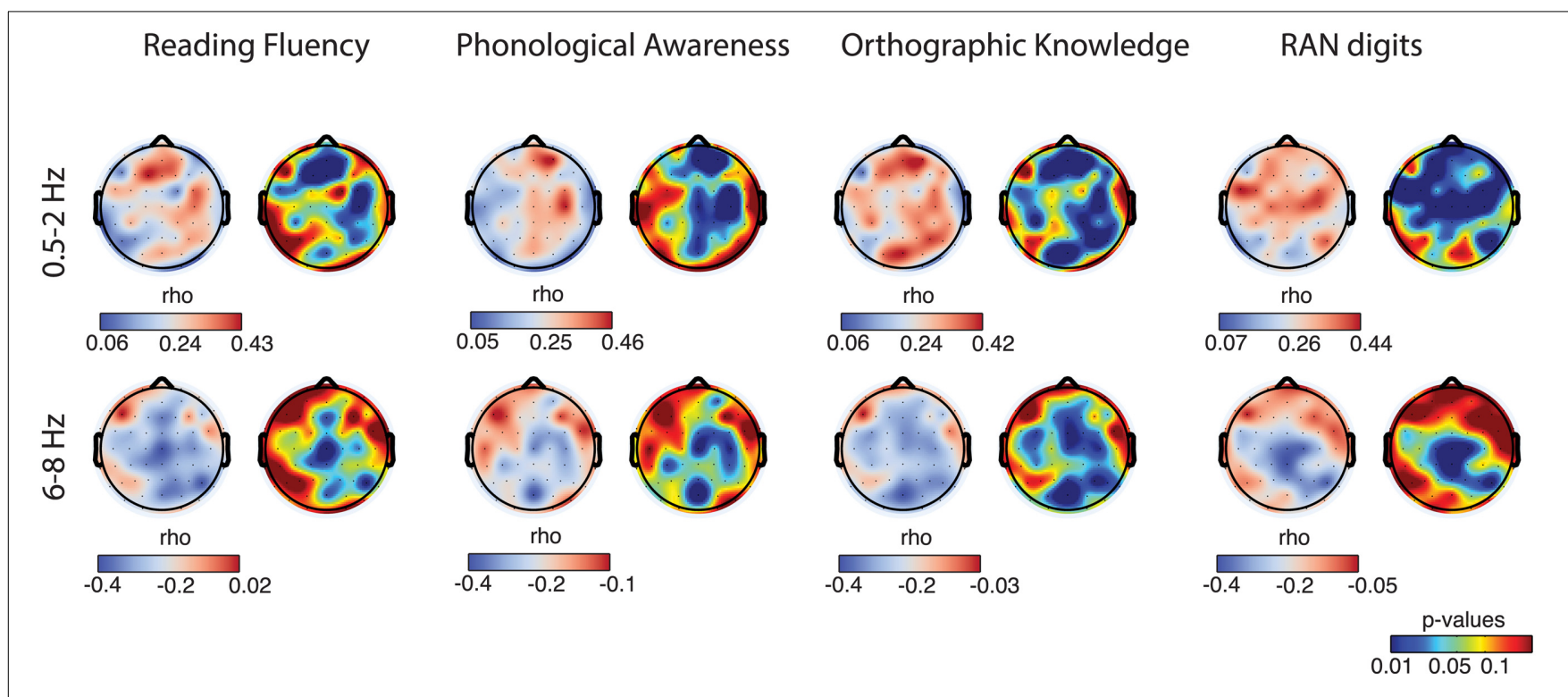

FIGURE 5 | Correlation topographies. For each behavioral task, correlations with delta-1 (first row) and alpha-1 (second row) relative amplitude of all the subjects are shown in the form of correlation coefficient ( $r$ ) topographies (cool-warm color map) and p-value topographies (jet color map).

signal analysis performed in our study did not include frequency components higher than $30 \mathrm{~Hz}$. Despite the differences in the cohort definition and in the EEG analysis, our results are congruent with the findings of Benasich et al. (2008) related to absence of statistical difference between control and at-risk fluent readers (comparable with $\mathrm{FH}+$ ) for spectral amplitude lower than $30 \mathrm{~Hz}$.

\section{CONCLUSIONS}

In conclusion, we showed that combining family-risk status assessment and resting-state EEG at preliterate age could provide preliminary indicators of future reading abilities. Specifically, our data suggest that delta and alpha oscillations are implicated with neurophysiological processes of importance for readingrelated disabilities later in childhood. In particular, we confirm the role of delta activity as a physiological index of abnormal cerebral maturation. Further investigations are required to better understand the functional significance and the underlying mechanisms governing the dynamics of these oscillations in developmental dyslexia. For example, in this study we did not account for special training in addition to schooling that some poor-reading children have followed, partially influencing their performances in the reading-related tasks measured at the middle of the third grade. In this regard, future studies may investigate whether reading-intervention programs (Connor et al., 2007)affect the dynamics of the brain as reflected in the resting-state EEG.

\section{ACKNOWLEDGMENTS}

We thank the Netherlands Organization for Scientific Research (NWO) for funding the Dutch Dyslexia Programme (1997-2012) under project number 200-62-304. We also thank all children and their parents for their dedicated participation in this longitudinal research program.

\section{REFERENCES}

Ahn, H., Prichep, L., John, E. R., Baird, H., Trepetin, M., and Kaye, H. (1980). Developmental equations reflect brain dysfunctions. Science 210, 1259-1262. doi: $10.1126 /$ science. 7434027

Araujo, S., Bramao, I., Faisca, L., Petersson, K. M., and Reis, A. (2012). Electrophysiological correlates of impaired reading in dyslexic pre-adolescent children. Brain Cogn. 79, 79-88. doi: 10.1016/j.bandc.2012.02.010

Babiloni, C., Stella, G., Buffo, P., Vecchio, F., Onorati, P., Muratori, C., et al. (2012). Cortical sources of resting state EEG rhythms are abnormal in dyslexic children. Clin. Neurophysiol. 123, 2384-2391. doi: 10.1016/j.clinph.2012.05.002

Benasich, A. A., Gou, Z., Choudhury, N., and Harris, K. D. (2008). Early cognitive and language skills are linked to resting frontal gamma power across the first 3 years. Behav. Brain Res. 195, 215-222. doi: 10.1016/j.bbr.2008.08.049

Brus, B. T., and Voeten, M. J. M. (1973). Een Minuut Test: Verantwoording En Handleiding (One Minute Test: Manual). Nijmegen: Berkhout.

Buchsbaum, B. (2001). Role of left posterior superior temporal gyrus in phonological processing for speech perception and production. Cogn. Sci. 25, 663-678. doi: 10.1207/s15516709 $\operatorname{cog} 2505 \_2$

Burton, M. W. (2009). Understanding the role of the prefrontal cortex in phonological processing. Clin. Linguist. Phon. 23, 180-195. doi: $10.1080 / 02699200802394963$

Chabot, R. J., di Michele, F., Prichep, L., and John, E. R. (2001). The clinical role of computerized EEG in the evaluation and treatment of learning and attention disorders in children and adolescents. J. Neuropsychiatry Clin. Neurosci. 13, 171-186. doi: 10.1176/appi.neuropsych.13.2.171

Choudhury, N., and Benasich, A. A. (2003). A family aggregation study: the influence of family history and other risk factors on language development. J. Speech Lang. Hear. Res. 46, 261. doi: 10.1044/1092-4388(2003/021)

Clarke, A. R., Barry, R. J., McCarthy, R., and Selikowitz, M. (2002). EEG analysis of children with attention-deficit/hyperactivity disorder and comorbid reading disabilities. J. Learn. Disabil. 35, 276-285. doi: 10.1177/0022219402035 00309

Colon, E. J., Notermans, S. L., De Weerd, J. P., and Kap, J. (1979). The discriminating role of EEG power spectra in dyslexic children. J. Neurol. 221, 257-262. doi: 10.1007/BF00314642

Connor, C. M., Morrison, F. J., Fishman, B. J., Schatschneider, C., and Underwood, P. (2007). Algorithm-guided individualized reading instruction. Science 315, 464-465. doi: 10.1126/science.1134513

De Jong, P. F., and Van der Leij, A. (2003). Developmental changes in the manifestation of a phonological deficit in dyslexic children learning to read a regular orthography. J. Educ. Psychol. 95, 22-40. doi: 10.1037/0022-0663.95.1.22 
Duffy, F. H., Denckla, M. B., Bartels, P. H., and Sandini, G. (1980). Dyslexia: regional differences in brain electrical activity by topographic mapping. Ann. Neurol. 7, 412-420. doi: 10.1002/ana.410070505

Eckert, M. A., Leonard, C. M., Wilke, M., Eckert, M., Richards, T., Richards, A., et al. (2005). Anatomical signatures of dyslexia in children: unique information from manual and voxel based morphometry brain measures. Cortex 41, 304-315. doi: 10.1016/S0010-9452(08)70268-5

Frank, R., and Hargreaves, R. (2003). Clinical biomarkers in drug discovery and development. Nat. Rev. Drug Discov. 2, 566-580. doi: 10.1038/nrd1130

Gasser, T., Verleger, R., Bacher, P., and Sroka, L. (1988). Development of the EEG of school-age children and adolescents. I. Analysis of band power. Electroencephalogr. Clin. Neurophysiol. 69, 91-99. doi: 10.1016/00134694(88)90204-0

Gou, Z., Choudhury, N., and Benasich, A. A. (2011). Resting frontal gamma power at 16, 24 and 36 months predicts individual differences in language and cognition at 4 and 5 years. Behav. Brain Res. 220, 263-270. doi: 10.1016/j.bbr.2011.01.048

Guttorm, T. K., Leppanen, P. H., Richardson, U., and Lyytinen, H. (2001). Eventrelated potentials and consonant differentiation in newborns with familial risk for dyslexia. J. Learn. Disabil. 34, 534-544. doi: 10.1177/0022219401034 00606

Hardstone, R., Poil, S.-S., Schiavone, G., Jansen, R., Nikulin, V. V., Mansvelder, H. D., et al. (2012). Detrended fluctuation analysis: a scale-free view on neuronal oscillations. Front. Physiol. 3:450. doi: 10.3389/fphys.2012.00450

Harmony, T., Marosi, E., Becker, J., Rodríguez, M., Reyes, A., Fernández, T., et al. (1995). Longitudinal quantitative EEG study of children with different performances on a reading-writing test. Electroencephalogr. Clin. Neurophysiol. 95, 426-433. doi: 10.1016/0013-4694(95)00135-2

Harmony, T., Marosi, E., Díaz de León, A. E., Becker, J., and Fernández, T. (1990). Effect of sex, psychosocial disadvantages and biological risk factors on EEG maturation. Electroencephalogr. Clin. Neurophysiol.75, 482-491. doi: 10.1016/0013-4694(90)90135-7

Horsley, T. M. (2005). Not all Dyslexics are Created Equal: Neurocognitive Evidence. Thesis, Vrije Universiteit, Amsterdam.

Humphrey, N., and Mullins, P. M. (2004). Self-concept and self-esteem in developmental dys- lexia. J. Res. Spec. Educ. Needs 2, 1-13. doi: 10.1111/j.14713802.2002.00163.x

John, E. R., Alan, H., Prichep, L., Trepetin, M., Brown, D., and Kaye, H. (1980) Developmental equations for the EEG. Science 210, 1255-1258. doi: 10.1126/science.7434026

Klimesch, W. (1999). EEG alpha and theta oscillations reflect cognitive and memory performance: a review and analysis. Brain Res. Rev. 29, 169-195. doi: 10.1016/S0165-0173(98)00056-3

Klimesch, W., Doppelmayr, M., Wimmer, H., Gruber, W., Röhm, D., Schwaiger, J., et al. (2001b). Alpha and beta band power changes in normal and dyslexic children. Clin. Neurophysiol. 112, 1186-11895. doi: 10.1016/S1388-2457(01) 00543-0

Klimesch, W., Doppelmayr, M., Wimmer, H., Schwaiger, J., Röhm, D., Gruber, W., et al. (2001a). Theta band power changes in normal and dyslexic children. Clin. Neurophysiol. 112, 1174-1185. doi: 10.1016/S1388-2457(01) 00545-4

Kort, W., Compaan, E. L., Bleichrodt, N., Resing, W. C. M., Schittekatte, M., Bosmans, M., et al. (2002). WISC-III NL. Handleiding. London: The Psychological Corporation.

Linkenkaer-Hansen, K., Smit, D. J. A., Barkil, A., van Beijsterveldt, T. E. M., Brussaard, A. B., Boomsma, D. I., et al. (2007). Genetic contributions to long-range temporal correlations in ongoing oscillations. J. Neurosci. 27, 13882-13889. doi: 10.1523/JNEUROSCI.3083-07.2007

Lyon, G. R., Shaywitz, S. E., and Shaywitz, B. A. (2003). A definition of dyslexia. Ann. Dyslexia 53, 1-14. doi: 10.1007/s11881-003-0001-9

Lyytinen, H., Aro, M., Eklund, K., Erskine, J., Guttorm, T., Laakso, M. L., et al. (2004). The development of children at familial risk for dyslexia: birth to early school age. Ann. Dyslexia 54, 184-220. doi: 10.1007/s11881-004-0010-3

Maris, E., and Oostenveld, R. (2007). Nonparametric statistical testing of EEG-and MEG-data. J. Neurosci. Methods 164, 177-190. doi: 10.1016/j.jneumeth.2007.03.024

Marshall, P. J., Bar-Haim, Y., and Fox, N. A. (2002). Development of the EEG from 5 months to 4 years of age. Clin. Neurophysiol. 113, 1199-1208. doi $10.1016 /$ S1388-2457(02)00163-3
Marshall, P. J., and Meltzoff, A. N. (2011). Neural mirroring systems: exploring the EEG mu rhythm in human infancy. Dev. Cogn. Neurosci. 1, 110-123. doi: 10.1016/j.dcn.2010.09.001

Maurer, U., Bucher, K., Brem, S., Benz, R., Kranz, F., Schulz, E., et al. (2009). Neurophysiology in preschool improves behavioral prediction of reading ability throughout primary school. Biol. Psychiatry 66, 341-348. doi: 10.1016/j.biopsych.2009.02.031

Maurer, U., Bucher, K., Brem, S., and Brandeis, D. (2003). Altered responses to tone and phoneme mismatch in kindergartners at familial dyslexia risk. Neuroreport 14, 2245-2250. doi: 10.1097/00001756-200312020-00022

Molfese, D. L. (2000). Predicting dyslexia at 8 years of age using neonatal brain responses. Brain Lang. 72, 238-245. doi: 10.1006/brln.2000.2287

Montez, T., Poil, S.-S., Jones, B. F., Manshanden, I., Verbunt, J. P. A., Van Dijk, B. W., et al. (2009). Altered temporal correlations in parietal alpha and prefrontal theta oscillations in early-stage Alzheimer disease. Proc. Natl. Acad. Sci. U.S.A. 106, 1614-1619. doi: 10.1073/pnas.0811699106

Nikulin, V. V., Jönsson, E. G., and Brismar, T. (2012). Attenuation of long-range temporal correlations in the amplitude dynamics of alpha and beta neuronal oscillations in patients with schizophrenia. Neuroimage 61, 162-169. doi: 10.1016/j.neuroimage.2012.03.008

Nolan, H., Whelan, R., and Reilly, R. B. (2010). FASTER: fully automated statistical thresholding for EEG artifact rejection. J. Neurosci. Methods 192, 152-162. doi: 10.1016/j.jneumeth.2010.07.015

Penolazzi, B., Spironelli, C., and Angrilli, A. (2008). Delta EEG activity as a marker of dysfunctional linguistic processing in developmental dyslexia. Psychophysiology 45, 1025-1033. doi: 10.1111/j.1469-8986.2008.00709.x

Pinkerton, F., Watson, D. R., and McClelland, R. J. (1989). A neurophysiological study of children with reading, writing and spelling difficulties. Dev. Med. Child Neurol. 31, 569-581. doi: 10.1111/j.1469-8749.1989.tb04042.x

Raschle, N. M., Chang, M., and Gaab, N. (2011). Structural brain alterations associated with dyslexia predate reading onset. Neuroimage 57, 742-749. doi: 10.1016/j.neuroimage.2010.09.055

Regtvoort, A. G. F. M., van Leeuwen, T. H., Stoel, R. D., and van der Leij, A. (2006). Efficiency of visual information processing in children at-risk for dyslexia: habituation of single-trial ERPs. Brain Lang. 98, 319-331. doi: 10.1016/j.bandl.2006.06.006

Richlan, F., Kronbichler, M., and Wimmer, H. (2009). Functional abnormalities in the dyslexic brain: a quantitative meta-analysis of neuroimaging studies. Hum. Brain Mapp. 30, 3299-3308. doi: 10.1002/hbm.20752

Rippon, G. (2000). Trait and state EEG indices of information processing in developmental dyslexia. Int. J. Psychophysiol. 36, 251-265. doi: 10.1016/S01678760(00)00075-1

Rumsey, J. M., Coppola, R., Denckla, M. B., Hamburger, S. D., and Kruesi, M. J. (1989). EEG spectra in severely dyslexic men: rest and word and design recognition. Electroencephalogr. Clin. Neurophysiol. 73, 30-40. doi: 10.1016/0013 4694(89)90017-5

Samuelsson, S. (2000). Converging evidence for the role of occipital regions in orthographic processing: a case of developmental surface dyslexia. Neuropsychologia 38, 351-362. doi: 10.1016/S0028-3932(99)00096-2

Scerri, T. S., and Schulte-Korne, G. (2010). Genetics of developmental dyslexia. Eur Child Adolesc. Psychiatry 19, 179-197. doi: 10.1007/s00787-009-0081-0

Schulte-Korne, G., and Bruder, J. (2010). Clinical neurophysiology of visual and auditory processing in dyslexia: a review. Clin. Neurophysiol. 121, 1794-1809. doi: 10.1016/j.clinph.2010.04.028

Sklar, B., Hanley, J., and Simmons, W. W. (1972). An EEG experiment aimed toward identifying dyslexic children. Nature 240, 414-416. doi: 10.1038/240414a0

Smit, D. J., de Geus, E. J. C., van de Nieuwenhuijzen, M. E., van Beijsterveldt, C. E. M., van Baal, G. C. M., Mansvelder, H. D., et al. (2011). Scale-free modulation of resting-state neuronal oscillations reflects prolonged brain maturation in humans. J. Neurosci. 31, 13128-13136. doi: 10.1523/JNEUROSCI.1678-11.2011

Snowling, M. J., Gallagher, A., and Frith, U. (2003). Family risk of dyslexia is continuous: individual differences in the precursors of reading skill. Child Dev. 74, 358-373. doi: 10.1111/1467-8624.7402003

Spironelli, C., and Angrilli, A. (2009). EEG delta band as a marker of brain damage in aphasic patients after recovery of language. Neuropsychologia 47, 988-994. doi: 10.1016/j.neuropsychologia.2008.10.019

Spironelli, C., and Angrilli, A. (2010). Developmental aspects of language lateralization in delta, theta, alpha and beta EEG bands. Biol. Psychol. 85, 258-267. doi: 10.1016/j.biopsycho.2010.07.011 
Spironelli, C., Angrilli, A., Calogero, A., and Stegagno, L. (2011). Delta EEG band as a marker of left hypofrontality for language in schizophrenia patients. Schizophr. Bull. 37, 757-767. doi: 10.1093/schbul/sbp145

Spironelli, C., Penolazzi, B., Vio, C., and Angrilli, A. (2006). Inverted EEG theta lateralization in dyslexic children during phonological processing. Neuropsychologia 44, 2814-2821. doi: 10.1016/j.neuropsychologia.2006.06.009

Spironelli, C., Penolazzi, B., Vio, C., and Angrilli, A. (2010). Cortical reorganization in dyslexic children after phonological training: evidence from early evoked potentials. Brain 133, 3385-3395. doi: 10.1093/brain/awq199

Stroganova, T. A., Orekhova, E. V., and Posikera, I. N. (1999). EEG alpha rhythm in infants. Clin. Neurophysiol. 110, 997-1012. doi: 10.1016/S1388-2457(98) 00009-1

Uterwijk, J. (2000). WAIS-III. Nederlandstalige Bewerking. Technische Handleiding. David Wechsler. Lisse: Swets Test Publishers.

van Bergen, E., de Jong, P. F., Maassen, B. A. M., Krikhaar, E., Plakas, A., and Van der Leij, A. (2013). IQ of four-year-olds who go on to develop dyslexia. J. Learn. Disabil. 47, 475-484. doi: 10.1177/0022219413479673

van Bergen, E., de Jong, P. F., Plakas, A., Maassen, B. A. M., and van der Leij, A. (2012). Child and parental literacy levels within families with a histroy of dyslexia. J. Child Psychol. Psychiatry 53, 28-36. doi: 10.1111/j.14697610.2011.02418.x

Van den Bos, K. P., lutje Spelberg, H. C., Scheepstra, A. J. M., and De Vries, J. R. (1994). De Klepel, vorm A en B. Een Test Voor de Leesvaardigheid Van Pseudowoorden. Verantwoording, Handleiding, Diagnostiek en Behandeling. Nijmegen: Berkhout. Test Materiaal.

Van Zuijen, T. L., Plakas, A., Maassen, B. A. M., Been, P., Maurits, N. M., Krikhaar, E., et al. (2012). Temporal auditory processing at 17 months of age is associated with preliterate language comprehension and later word reading fluency: an ERP study. Neurosci. Lett. 528, 31-35. doi: 10.1016/j.neulet.2012. 08.058

Van Zuijen, T. L., Plakas, A., Maassen, B. A., Maurits, N. M., and Leij, A. (2013). Infant ERPs separate children at risk of dyslexia who become good readers from those who become poor readers. Dev. Sci. 16, 554-563. doi: 10.1111/desc. 12049
Vellutino, F. R., Fletcher, J. M., Snowling, M. J., and Scanlon, D. M. (2004). Specific reading disability (dyslexia): what have we learned in the past four decades? J. Child Psychol. Psychiatry 45, 2-40. doi: 10.1046/j.0021-9630.2003.00305.x

Vellutino, F. R., and Scanlon, D. M. (1987). Phonological coding, phonological awareness, and reading ability: evidence from a longitudinal and experimental study. Merrill Palmer Q. 33, 321-363.

Verhoeven, L. (1995). Drie-Minuten-Toets (DMT): Handleiding. Arnhem: Cito.

Wechsler, D. (1997). Wechsler Adult Intelligence Scale-Third Edition. San Antonio, TX: The Psychological Corporation.

Wechsler, D. A. (1992). Wechsler Intelligence Scale for Children-III. San Antonio, TX: The Psychological Corporation.

Whitford, T. J., Rennie, C. J., Grieve, S. M., Clark, C. R., Gordon, E., and Williams, L. M. (2007). Brain maturation in adolescence: concurrent changes in neuroanatomy and neurophysiology. Hum. Brain Mapp. 28, 228-237. doi: $10.1002 / \mathrm{hbm} .20273$

Conflict of Interest Statement: The authors declare that the research was conducted in the absence of any commercial or financial relationships that could be construed as a potential conflict of interest.

Received: 25 May 2014; accepted: 01 September 2014; published online: 19 September 2014.

Citation: Schiavone G, Linkenkaer-Hansen K, Maurits NM, Plakas A, Maassen BAM, Mansvelder HD, van der Leij A and van Zuijen TL (2014) Preliteracy signatures of poor-reading abilities in resting-state EEG. Front. Hum. Neurosci. 8:735. doi: 10.3389/ fnhum.2014.00735

This article was submitted to the journal Frontiers in Human Neuroscience.

Copyright (C) 2014 Schiavone, Linkenkaer-Hansen, Maurits, Plakas, Maassen, Mansvelder, van der Leij and van Zuijen. This is an open-access article distributed under the terms of the Creative Commons Attribution License (CC BY). The use, distribution or reproduction in other forums is permitted, provided the original author(s) or licensor are credited and that the original publication in this journal is cited, in accordance with accepted academic practice. No use, distribution or reproduction is permitted which does not comply with these terms. 